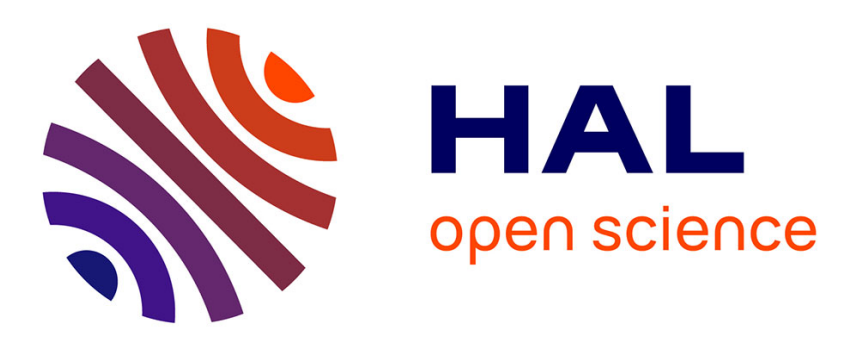

\title{
Process formation results from the imbalance between motor-mediated forces
}

\author{
Lotfi Ferhat, Guillaume Rami, Igor Medina, Yehezkel Ben-Ari, Alfonso
}

Represa

\section{> To cite this version:}

Lotfi Ferhat, Guillaume Rami, Igor Medina, Yehezkel Ben-Ari, Alfonso Represa. Process formation results from the imbalance between motor-mediated forces. Journal of Cell Science, 2001, 114 (Pt 21), pp.3899-3904. inserm-00484926

\section{HAL Id: inserm-00484926 https://www.hal.inserm.fr/inserm-00484926}

Submitted on 19 May 2010

HAL is a multi-disciplinary open access archive for the deposit and dissemination of scientific research documents, whether they are published or not. The documents may come from teaching and research institutions in France or abroad, or from public or private research centers.
L'archive ouverte pluridisciplinaire HAL, est destinée au dépôt et à la diffusion de documents scientifiques de niveau recherche, publiés ou non, émanant des établissements d'enseignement et de recherche français ou étrangers, des laboratoires publics ou privés. 


\title{
Process formation results from the imbalance between motor-mediated forces
}

\author{
Lotfi Ferhat*, Guillaume Rami, Igor Medina, Yehezkel Ben-Ari and Alfonso Represa \\ INMED/INSERM U29, 163 rue de Luminy, BP 13, 13273 Marseille Cedex 09, France \\ *Author for correspondence (e-mail: ferhat@inmed.univ-mrs) \\ Accepted 26 July 2001 \\ Journal of Cell Science 114, 3899-3904 (2001) @ The Company of Biologists Ltd
}

\section{SUMMARY}

Several reports have suggested that neurite outgrowth is mediated by opposing forces generated on microtubules and microfilaments but the molecular basis underlying these forces have not been determined. Here, we show that in non-neuronal cell lines, the inhibition of actomyosin activity by acidic calponin promotes the formation of processes. This effect is blocked by inhibition of the motor activity of cytoplasmic dynein. Therefore, neurite formation is due to an imbalance between tensile and compressive forces mediated by myosins and dyneins, respectively. We propose a mechanism that involves the motor-mediated forces in a tight regulation of the process formation.

Key words: Microtubule, Microfilament, Motor proteins, Acidic calponin, Dynamitin

\section{INTRODUCTION}

The development of axons and dendrites is critical for the establishment of synaptic connections between neurons in order to form functional networks but the mechanisms that support formation of these processes in developing neurons are not completely understood. Several reports have suggested that neurite outgrowth implicates the interaction between microfilaments and microtubules. These cytoskeletal components produce opposing forces: tension produced by microfilaments of the cell cortex (Bray et al., 1986; Zheng et al., 1991; Edson et al., 1993) and compression generated by microtubule bundles in the cytoplasm (Letourneau et al., 1987; Dennerll et al., 1989; Edson et al., 1993). However, the molecular basis for the generation of such forces needs to be investigated. The cytoplasm is rich in molecular motor proteins such as dynein and myosin, which appear to be important in generating forces on microtubules and microfilaments (Carminati and Stearns, 1997; Busson et al., 1998; Evans et al., 1998; Inoue et al., 1998). The first evidence of motor driven forces in neurite outgrowth has emerged from recent studies by Ahmad and collaborators (Baas and Ahmad, 2001). Indeed, the formation of neurites seems to depend on the forces generated upon the microtubules by the motor protein dynein (Ahmad et al., 1998). Moreover, Ahmad and colleagues have shown that the inhibition of the motor activity of dynein causes the axon to retract and that this effect is prevented if myosins are inhibited (Ahmad et al., 2000).

From these observations, we postulate that process formation results from the imbalance between tensile and compressive forces mediated by myosins and dyneins, respectively. To test this hypothesis, we transfected non-neuronal cells that do not develop processes in control conditions with acidic calponin (ac.CaP), an actin-binding protein known to inhibit the actomyosin activity (Gimona and Small, 1996; Winder and Walsh, 1996; Winder et al., 1998) and/or dynamitin, which when present at abnormally high levels results in an immediate cessation of dynein activity (Echeverri et al., 1996; Wittmann and Hyman, 1999). We report that the overexpression of ac.CaP induces the formation of cell processes and that this effect is blocked by the overexpression of dynamitin. Our results provide evidence that the formation of processes, previously shown to be induced by a depolymerisation of microfilaments (Letourneau et al., 1987; Edson et al., 1993; Ferhat et al., 1996b; Ferhat et al., 1998; Meberg and Bamburg, 2000), is also produced by an inhibition of myosin-mediated forces. Our observations strongly support the idea that the formation of processes is a result of antagonistic forces. In addition, consistent with recent observations on primary neurons (Ahmad et al., 2000), our results indicate that these antagonistic forces are generated by motor proteins: tensile forces generated by myosins and compressive forces generated by dyneins. We conclude that the imbalance between myosin and dyneinmediated forces determines whether a neurite forms and elongates or whether it retracts.

\section{MATERIALS AND METHODS}

\section{RNA purification and reverse transcription}

Total RNA was prepared from the hippocampi of newborn or adult rats by the acid guanidinium thiocyanate-phenol-chloroform extraction method (Chomczynski and Sacchi, 1987). $1 \mu \mathrm{g}$ of total RNA samples was reverse transcribed using ThermoScript RT-PCR System from Gibco BRL. The cDNA synthesis reactions were performed at $25^{\circ} \mathrm{C}$ for 10 minutes and at $55^{\circ} \mathrm{C}$ for 60 minutes. To terminate the reactions samples were incubated for 5 minutes at $85^{\circ} \mathrm{C}$. To remove the RNA prior to PCR, the cDNA samples were treated with $1 \mu \mathrm{l}$ of RNase $\mathrm{H}$ at $37^{\circ} \mathrm{C}$ for 20 minutes. Finally, the samples were diluted five times with water.

PCR, analysis of amplified PCR products and cloning

cDNAs encoding the full length of ac.CaP and dynamitin were 
amplified by PCR from P0 and adult rat hippocampal RNA, respectively, using the ThermoScript RT-PCR System, with forward primers containing an $E c o \mathrm{RI}$ and reverse primers containing a BamHI restriction sites. Ac.CaP primers were EcoRI calp: tatatagaattc CAGCCATGACCCACTTAACAAGGGCCCT and BamHI calp: atatatggatccce GTAATCGATGCCCTGGTCGTCAC. Dynamitin primers were EcoRI dyn: tatatagaattccagaa ATGGCGGACCCTAAATACG and BamHI dyn: atatatggatccc CTTTCCCAGCTTCTTCATCCGTTC. All the primers were selected in the rat cDNA sequences (Ferhat et al., 1996a; Echeverri et al., 1996).

PCR reactions were carried out in a programmable heating block (GeneAmp PCR System 9700). Following the gel analysis, the PCR products were purified using Qiagen spin columns. Finally, a PCR fragment encoding the full length ac. CaP protein was inserted into the mammalian expression vectors pDsRed1-N1 and pEGFP-N1 (Clontech) and the ones encoding the full length dynamitin and ac.CaP $\Delta$ proteins were inserted into pEGFP-N1. The vectors pDsRed1-N1 and pEGFP-N1 encode for Red Fluorescent Protein (RFP) and Green Fluorescent Protein (GFP), respectively. The obtained constructs were subsequently fully sequenced in order to verify the integrity of the fusion protein (ac.CaP-RFP/-GFP, dynamitin-GFP and ac.CaP $\Delta$-GFP).

\section{Cell line and transfection}

Human embryonic kidney (HEK 293) cells from the American Type Tissue Culture Collection (ATCC) were grown in MEM (minimum essential medium, Gibco BRL), supplemented with $10 \%$ fetal bovine serum (FBS, Gibco BRL), 2 mM glutamine (Gibco BRL), $100 \mathrm{IU} / \mathrm{ml}$ penicillin and $100 \mathrm{mg} / \mathrm{ml}$ streptomycin (Sigma). Transfections were performed using Lipofectamine Plus according to the manufacturer's protocol (Gibco BRL). Twenty-four hours after transfection, cells were fixed with $4 \%$ paraformaldehyde in phosphate buffered saline (PBS, pH 7.2) for 20 minutes at room temperature (RT).

Quantitative analyses of the number of transfected cells were performed using a fluorescence microscope with a $20 \times$ objective. Twenty fields per coverslip per experiment $(n=7)$ were analysed. Data were expressed as the mean percentage of total cells per experiment \pm s.e.m.

\section{Immunofluorescence}

Fixed cells were permeabilized with $0.5 \%$ Triton X-100 for 10 minutes and incubated overnight at $4{ }^{\circ} \mathrm{C}$ with mouse monoclonal antibody directed against $\beta$-tubulin (1/100, Sigma). Tubulin staining was revealed with the fluorescein (FITC)-conjugated goat anti-mouse (1/200, Jackson Immunoresearch). Before the actin staining, the cells were permeabilized with $0.1 \%$ Triton $\mathrm{X}-100$ for 5 minutes and exposed for 2 hours at RT to 0.5 unit per coverslip of Texas Red-X phalloidin (Molecular Probes). Cells were then mounted with Fluoromount G (Southern Biotechnology Associates) and analysed using Zeiss LSM-410 laser scanning microscope.

\section{RESULTS}

\section{Overexpression of acidic calponin induces process formation in non-neuronal cells}

Fig. 1 shows the effect of overexpression of ac.CaP on the cellular morphology of HEK 293 cells. Cells transfected with RFP used as control (Fig. 1A) displayed a diffuse staining pattern in the cytoplasm as well as in the nucleus, but the staining in the latter was stronger. Furthermore, these cells did not show any morphological changes compared with untransfected cells (data not shown). In contrast to RFP, HEK 293 transfected with ac.CaP-RFP displayed striking morphological changes (observed in $38 \pm 3 \%$ ) characterised by

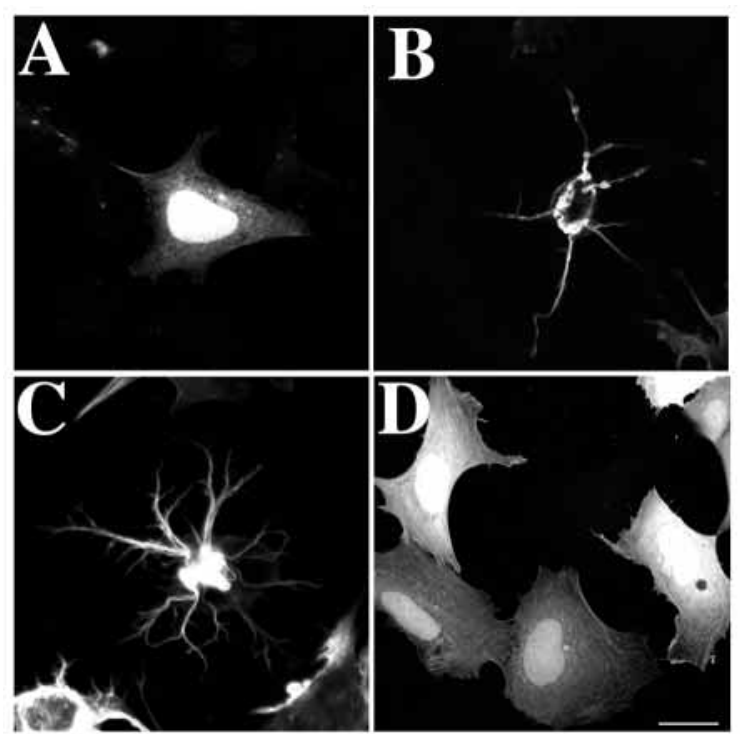

Fig. 1. Transfection of cultured HEK 293 cells. (A) A cell transfected with RFP. Diffuse RFP protein is observed in the cytoplasm and the nucleus but the staining in the latter is stronger. $(\mathrm{B}, \mathrm{C})$ Two examples of cells transfected with ac.CaP-RFP. These ac.CaP-RFP cells display striking morphological changes compared with RFP-transfected cells. (D) A cell transfected with ac.CaP $\Delta$-GFP that does not have the actin-binding domains. The staining observed is diffuse in the cytoplasm and the nucleus. The ac.CaP $\Delta$-GFPtransfected cells did not display any morphological change compared with RFP-transfected cells. Bar, $15 \mu \mathrm{m}$.

the formation of several processes (Fig. 1B,C) frequently branched (Fig. 1C). The shape of the cell body was rather round and its size was significantly reduced. In addition, ac.CaP-RFP was absent from the nucleus but it appeared to form thick twisted bundles within the cytoplasm, from which filaments radiate to project into the extensions (Fig. 1B,C). Similar observations were obtained using ac.CaP-GFP (Fig. 2) as well as on CHO-K1 cells (data not shown). In order to assess that ac.CaP-RFP/GFP exerts its effect via its interaction with the actomyosin system, we generated a mutant ac.CaP-GFP without actin-binding domains (amino-acid residues 142-272). As with RFP, cells transfected with ac.CaP $\Delta$-GFP displayed a diffuse staining pattern in the cytoplasm as well as in the nucleus. In contrast to ac.CaP-GFP, ac.CaP $\Delta$-GFP, which do not have the actin-binding domains, did not exhibit any morphological changes (Fig. 1D). This observation reinforces the idea that ac.CaP acts through the inhibition of the actomyosin system to induce processes in non neuronal cells.

\section{Acidic calponin induces a reorganisation of microfilament arrays}

It has been shown that ac.CaP binds microfilaments (Applegate et al., 1994). To verify that overexpressed ac.CaP-GFP still binds to microfilaments, we visualised them using Texas Red$\mathrm{X}$ phalloidin. In this set of experiments we chose ac.CaP-GFP instead of ac.CaP-RFP because of a better visualisation of microfilaments staining using Texas Red-X phalloidin. These studies also allowed the evaluation of the effects of ac.CaPGFP on the microfilaments organisation.

In control cells, Texas Red-X phalloidin detected the presence of actin stress fibres that cross over the cytoplasm and 
bundles of actin filaments beneath the plasma membrane (Fig. 2A). In all transfected cells (Fig. 2B-D), ac.CaP-GFP (Fig. 2C) was mainly co-distributed with microfilaments (Fig. 2B,D, yellow) suggesting that the majority of ac.CaP-GFP effectively bound microfilaments. Furthermore, our results show that in the transfected cells there was a striking microfilament reorganisation (Fig. 2B,D): twisted bundles of microfilaments were observed in the cell body. A few actin bundles that originate from this central core projected within the extensions. In addition, these cells had lost both actin stress fibres and cortical actin bundles.

\section{Acidic calponin induces a reorganisation of microtubule arrays}

Using $\beta$-tubulin antibodies, we investigated the effects of ac.CaP-RFP on microtubule organisation (Fig. 3). In control cells (Fig. 3A-C), microtubules emerging from a centrosomal organising centre next to the nucleus radiated through the cytoplasm as individual filaments (Fig. 3B) until they were stopped by the presence of cortical actin filaments (Fig. 3A,C). By contrast, ac.CaP-RFP-transfected cells (Fig. 3D) displayed a clear-cut reorganisation of microtubules (Fig. 3E). Thick bundles of microtubules were observed within the cell bodies and extensions. In all transfected cells, the ac.CaP-RFP (in red) was not co-localised with microtubules (in green) but rather was adjacent (Fig. 3F). In addition, the ac.CaP/microfilaments bundles were detached from the cell cortex and localised in the core of the cytoplasm so that microtubules distributed at the cell periphery (Fig. 3F). We also noticed that in many cases, ac.CaP filaments/microfilaments (Fig. 3G,I, red) and microtubules (Fig. 3H,I, green) clearly form 'curves' within the processes (see arrows). This wavy pattern of microfilaments and microtubules might be a consequence of the forces generated by motor proteins and bore by these two cytoskeletal elements.

\section{Process formation induced by acidic calponin depends on dynein activity}

There is much evidence showing that dynein motor may generate forces between microtubules and microfilaments in mitotic cells and postmitotic neurons (Baas and Ahmad, 2000). In mitotic cells, it has been suggested that dynein-mediated forces are required for positioning microtubules and centrosomes during interphase anaphase (Koonce et al., 1999; Ma et al., 1999) and separating duplicated centrosomes during prophase and anaphase (Carminati and Stearns, 1997; Busson et al., 1998; Inoue et al., 1998; Gonczy et al., 1999). In postmitotic neurons, these same forces are essential for the transport of microtubules from the centrosome into the axon and this transport is required in axon outgrowth (Ahmad et al., 1998). Here we tested the hypothesis that process formation induced by the overexpression of ac. $\mathrm{CaP}$ depends on the dynein activity. If this is correct, the effects of ac.CaP should be blocked by the inhibition of dynein activity. For this purpose, we simultaneously overexpressed ac.CaP-RFP and dynamitin-GFP in HEK 293 cells. Indeed, when present at abnormally high levels, dynamitin is known to inhibit dynein activity. As we have shown above, the overexpression of ac.CaP-RFP induced the formation of several processes (Fig. 4A,C, red). By contrast, dynamitin-GFP-transfected cells did not show any significant morphological change and never displayed extensions (Fig. 4B,C, green). As described by Burkhardt et al., dynamitin-GFP protein was diffusely distributed in the cytoplasm and was absent from the nucleus (Fig. 4B) (Burkhardt et al., 1997). Double-transfected cells overexpressing both ac.CaP-RFP (Fig. 4D) and dynamitinGFP (Fig. 4E) did not develop significant processes (Fig. 4F). The size of double-transfected cells was significantly reduced (Fig. 4F) compared with cells expressing either ac.CaP-RFP or dynamitin-GFP (Fig. 4C). Similar observations were obtained using CHO-K1 cells (data not shown). We cannot completely exclude that dynamitin effects are related to the alteration of the Golgi apparatus (Burkhardt et al., 1997; Ahmad et al., 1998). However, our data, in agreement with previous observations (Ahmad et al., 2000), strongly support the notion that dynein-mediated forces between microtubules and microfilaments play a key role in process formation.

\section{DISCUSSION}

The present data show that the overexpression of ac.CaP in non-neuronal cells induces the formation of processes and that this effect depends on dynein activity.

Previous data have demonstrated that the formation of neurites and their elongation are induced by the depolymerisation of microfilaments using destabilising agents such as cytochalasins (Marsh and Letourneau, 1984; Bentley and Toroian-Raymond, 1986; Letourneau et al., 1987; Ferhat et al., 1996b; Ferhat et al., 1998). Consistent with these observations, it has more recently been reported that the depletion of microfilaments by the overexpression of endogenous factors such as actin depolymerising factor (ADF) also promotes neurite elongation (Meberg and Bamburg, 2000). By contrast, our results suggest that process formation induced by ac.CaP did not require a depolymerisation mechanism. Several lines of evidence reinforce this idea. First, cytochalasin treatment of control HEK 293 cells does not induce process formation (Ferhat et al., 1996b). Second, the main in vitro effect of calponin family is to inhibit the actomyosin activity (Gimona and Small, 1996; Winder and Walsh, 1996; Winder et al., 1998). Third, biochemical studies have shown that calponins also stimulate actin polymerisation, and bundling and stabilisation of F-actin filaments (Kake et al., 1995; Kolakowski et al., 1995). In agreement with these in vitro effects our present data show that ac.CaP-transfected cells display a more bundled organisation of actin filaments compared with control cells. Thus we suggest that the formation of processes induced by ac. CaP is due to the inhibition of actomyosin-mediated forces rather than to the depletion of the microfilaments array. Based on all these observations we propose that, to generate processes, cells can use two independent mechanisms in parallel: disassembly of microfilaments and inhibition of actomyosin activity.

In non-neuronal cells, the microtubules radiate through the cytoplasm as individual filaments until they are stopped by a dense network of actin filaments of the cell cortex. This cortical cytoskeleton has been suggested to act as a tensile envelope maintaining the shape of the cell (Bray and White, 1988; Janmey, 1991). In addition, physical studies indicated that actin filaments are more resistant to deformation than microtubules (Janmey et al., 1991). By contrast, when ac.CaP is 

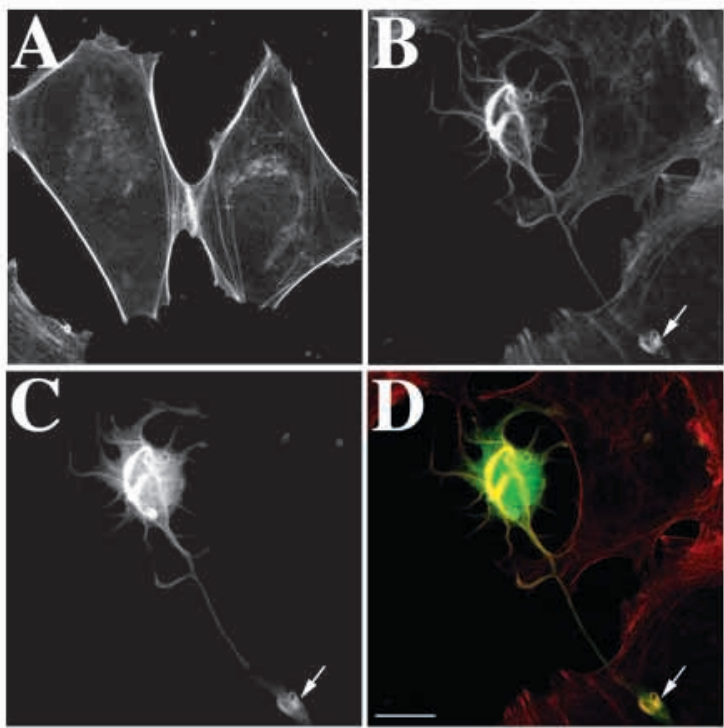

Fig. 2. Microfilament organisation in HEK 293 cells transfected with acidic calponin. Panels A and B illustrate untransfected control cells and ac.CaP-GFP-transfected cells stained with Texas Red-X phalloidin, respectively. The organisation of microfilaments is dramatically altered in ac.CaP-GFP cells (C). Indeed, microfilaments are thicker and display a twisted organisation compared with control cells. Panel D corresponds to the overlay of B and C. The yellow color indicates that ac.CaP-GFP mainly binds to F-actin. Note the accumulation of calponin and microfilaments within the tip of the process (see arrows). Bar, $15 \mu \mathrm{m}$.

overexpressed in HEK 293 cells, the organisation of these cytoskeleton components is strikingly altered. Indeed, in these cells, microtubules were localised at the cell periphery while microfilaments were detached from the cell cortex and accumulated within the cytoplasm. Therefore, the microfilament reorganisation observed in ac.CaP cells facilitates the microtubule bundles to pull out processes. One possible explanation of the microfilament reorganisation relates to the inability of myosins to generate forces required for the transport of microfilaments into the periphery. Indeed, it is now well established that motor proteins, known to convert the chemical energy released by nucleotide hydrolysis directly into movement, are capable not only of transporting organelles along microtubules or microfilaments but also of transporting and organising the cytoskeleton components themselves. Specifically, it has been shown that myosins are involved in the organisation and transport of microfilaments (Evans et al., 1998; Wu et al., 2000). Thus, the processes induced by ac.CaP may be mediated by the attenuation of myosin forces generated on microfilament arrays.

Our data show that the overexpression of ac.CaP is associated with the formation of processes containing microtubules. These processes cannot be the result of the assembly properties of microtubules since their formation depends on the dynein activity. It has been suggested that dynein drives microtubules down to the axon by generating forces upon the microfilament array (Ahmad et al., 1998; Baas, 1999; Baas and Ahmad, 2001). Thus we propose that the processes induced by the ac.CaP are mediated by the organisation and the transport of microtubules by dyneinmediated forces. This strongly supports the idea that the
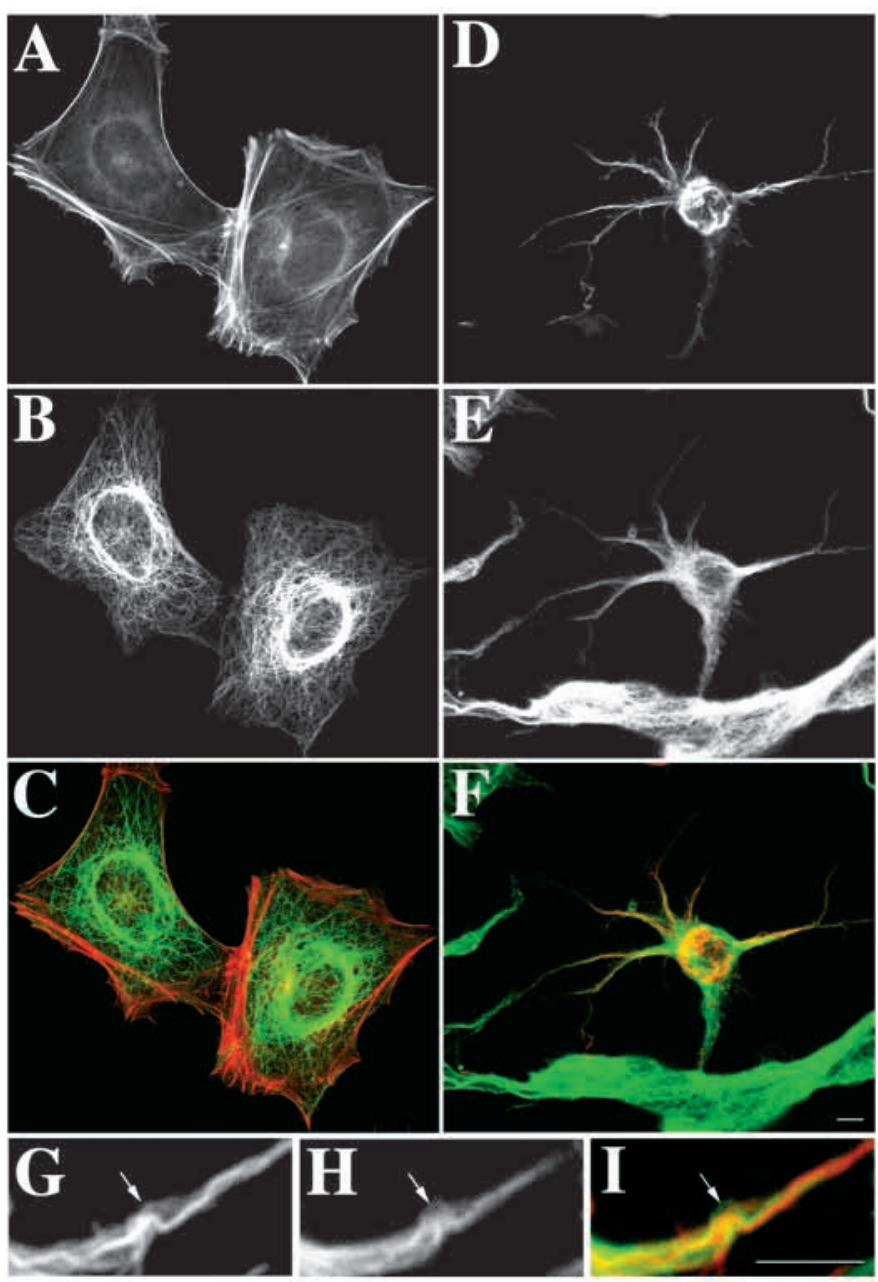

Fig. 3. Microtubule organisation in HEK 293 cells transfected with acidic calponin. Panels A and B illustrate the same control cells double stained with Texas Red-X phalloidin and $\beta$-tubulin antibodies, respectively. Panel $\mathrm{C}$ corresponds to the overlay of A and B. It shows that microtubules radiated through the cytoplasm as individual filaments until stopped by the cortical actin filaments. Panel D shows an ac.CaP-RFP-transfected cell. Panel E illustrates the same cell counterstained with $\beta$-tubulin antibodies. Panel F corresponds to the overlay of D and E. It shows that ac.CaP-RFPtransfected cells displayed a clear-cut reorganisation of microtubules compared with control cells. Thick bundles of microtubules were observed within the cell bodies and extensions. Moreover, the ac.CaP-RFP (in red) was not co-distributed with microtubules (in green) but rather adjacent. Panel G shows a process of an ac.CaPRFP cell. Panel $\mathrm{H}$ illustrate the same process counterstained with $\beta$ tubulin antibodies. Panel I corresponds to the overlay of $\mathrm{G}$ and $\mathrm{H}$. It shows that ac.CaP filaments/microfilaments (in red) and microtubules (in green) clearly form 'curves' within this process (see arrows). Bar, $15 \mu \mathrm{m}$.

formation of processes is a result of antagonistic forces (Letourneau et al., 1987; Bray et al., 1986; Dennerll et al., 1989; Zheng et al., 1991; Edson et al., 1993) that are generated by motor proteins (Ahmad et al., 2000; Baas and Ahmad, 2001): tensile forces generated by myosins and compressive forces generated by dyneins. We propose that it is the imbalance between these forces that determines whether a process forms and elongates or whether it retracts. According to this model, a decrease in the tensile forces generated by 


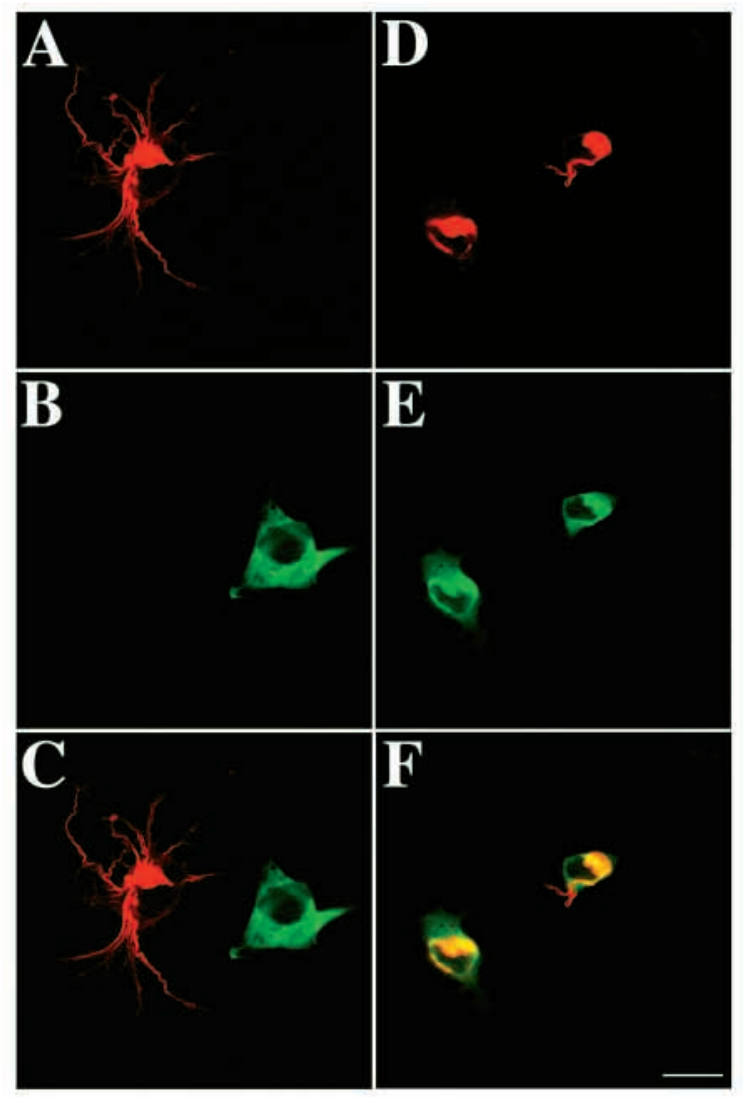

Fig. 4. Inhibition of dynein activity blocks process formation induced by acidic calponin. Panel A shows a cell that overexpresses ac.CaPRFP and extends several processes. Panel B shows that a cell overexpressing dynamitin-GFP does not display any morphological change. The Red color corresponds to cells expressing ac.CaP-RFP, the green color indicates cells overexpressing dynamitin-GFP. Panel $\mathrm{C}$ corresponds to the overlay of panels $\mathrm{A}$ and $\mathrm{B}$. Panels D-F illustrate the same cells double transfected with ac.CaP-RFP (in red) and dynamitin-GFP (in green). Panel F corresponds to the overlay of panels $\mathrm{D}$ and $\mathrm{E}$. It shows that all cells overexpressing both ac.CaPRFP and dynamitin-GFP do not develop processes. Note also that cells double transfected with ac.CaP-RFP and dynamitin-GFP are smaller than cells expressing either ac.CaP-RFP (A) or dynamitinGFP (B). Bar, $30 \mu \mathrm{m}$.

myosins leads dyneins to induce process formation and elongatation. By contrast, an attenuation of the compressive forces generated by dyneins leads myosins to cause the process to retract.

Our observations in non-neuronal cells probably reflect the mechanisms that can be used by neurons during their development. Interestingly, we have shown that ac.CaP is a variant particularly enriched in neurons during early development (Ferhat et al., 1996b), where it co-localises with microfilaments in cell bodies and growth cones (Plantier et al., 1999). Preliminary data show that overexpression of acidic calponin in cultured hippocampal neurons induces a dramatic increase of neuritic branches (L. Ferhat et al., unpublished). These results suggest that during neuronal development, neurons generate their neurites using ac. $\mathrm{CaP}$ as an endogenous regulator that may modulate the actomyosin system. Ahmad et al. showed that inhibition of the motor activity of dynein induces the axon to retract and that this effect depends on myosin-mediated forces (Ahmad et al., 2000). Our observations indicate that the same motor-mediated forces are also involved in the formation, and probably in the elongation of neurites. Thus, the model of motor-generated forces proposed for non-neuronal cells may be extended to neurons.

In conclusion, we propose that the motor-mediated forces are one of the mechanisms by which microfilaments and microtubules are integrated in order to modulate the cell morphology.

We thank Djaffar Boussa and Monique Esclapez for critical reading the manuscript. L.F. is recipient of a 'poste vert' from INSERM.

\section{REFERENCES}

Ahmad, F. J., Echeverri, C. J., Vallee, R. B. and Baas, P. W. (1998). Cytoplasmic dynein and dynactin are required for the transport of microtubules into the axon. J. Cell Biol. 140, 391-401.

Ahmad, F. J., Hughey, J., Wittmann, T., Hyman, A., Greaser, M. and Baas, P. W. (2000). Motor proteins regulate force interactions between microtubules and microfilaments in the axon. Nat. Cell Biol. 2, 276-280.

Applegate, D., Feng, W., Green, R. S. and Taubman, M. B. (1994). Cloning and expression of a novel acidic calponin isoform from rat aortic vascular smooth muscle. J. Biol. Chem. 269, 10683-10690.

Baas, P. W. (1999). Microtubules and neuronal polarity: lessons from mitosis. Neuron 22, 23-31.

Baas, P. W. and Ahmad F. J. (2001). Force generation by cytoskeletal motor proteins as a regulator of axonal elongation and retraction. Trends Cell Biol. 11, 244-249.

Bentley, D. and Toroian-Raymond, A. (1986). Disoriented pathfinding by pioneer neuron growth cones deprived of filopodia by cytochalasin treatment. Nature 323, 712-715.

Bray, D., Heath, J. and Moss, D. (1986). The membrane-associated 'cortex' of animal cells: its structure and mechanical properties. J. Cell Sci. Suppl. 4, 71-88.

Bray, D. and White, J. G. (1988). Cortical flow in animal cells. Science 239, 883-888.

Burkhardt, J. K., Echeverri, C. J., Nilsson, T. and Vallee, R. B. (1997). Overexpression of the dynamitin (p50) subunit of the dynactin complex disrupts dynein-dependent maintenance of membrane organelle distribution. J. Cell Biol. 139, 469-484.

Busson, S., Dujardin, D., Moreau, A., Dompierre, J. and De Mey, J. R. (1998). Dynein and dynactin are localized to astral microtubules and at cortical sites in mitotic epithelial cells. Curr. Biol. 8, 541-544.

Carminati, J. L. and Stearns, T. (1997). Microtubules orient the mitotic spindle in yeast through dynein-dependent interactions with the cell cortex. J. Cell Biol. 138, 629-641.

Chomczynski, P. and Sacchi, N. (1987). Single-step method of RNA isolation by acid guanidinium thiocyanate-phenol-chloroform extraction. Anal. Biochem. 162, 156-159.

Dennerll, T. J., Lamoureux, P., Buxbaum, R. E. and Heidemann, S. R. (1989). The cytomechanics of axonal elongation and retraction. J. Cell Biol. 109, 3073-3083.

Echeverri, C. J., Paschal, B. M., Vaughan, K. T. and Vallee, R. B. (1996). Molecular characterization of the 50-kD subunit of dynactin reveals function for the complex in chromosome alignment and spindle organization during mitosis. Cell Biol. 132, 617-633.

Edson, K., Weisshaar, B. and Matus, A. (1993). Actin depolymerisation induces process formation on MAP2-transfected non-neuronal cells. Development. 117, 689-700.

Evans, L. L., Lee, A. J., Bridgman, P. C. and Mooseker, M. S. (1998). Vesicle-associated brain myosin- $\mathrm{V}$ can be activated to catalyze actin-based transport. J. Cell Sci. 111, 2055-2066.

Ferhat, L., Charton, G., Represa, A., Ben-Ari, Y., der Terrossian, E. and Khrestchatisky, M. (1996a). Acidic calponin cloned from neural cells is differentially expressed during rat brain development. Eur. J. Neurosci. 8, 1501-1509.

Ferhat, L., Cook, C., Kuriyama, R. and Baas, P. W. (1998). The nuclear/mitotic apparatus protein NuMA is a component of the somatodendritic microtubule arrays of the neuron. J. Neurocytol. 27, 887-899. 
Ferhat, L., Represa, A., Bernard, A., Ben-Ari, Y. and Khrestchatisky, M. (1996b). MAP2d promotes bundling and stabilization of both microtubules and microfilaments. J. Cell Sci. 109, 1095-1103.

Gimona, M. and Small, J. V. (1996). Calponin. In Biochemistry of Smooth Muscle Contraction (ed. M. Barany), pp. 91-103. San Diego, CA: Academic Press.

Gonczy, P., Pichler, S., Kirkham, M. and Hyman, A. A. (1999). Cytoplasmic dynein is required for distinct aspects of MTOC positioning, including centrosome separation, in the one cell stage Caenorhabditis elegans embryo. J. Cell Biol. 147, 135-150.

Inoue, S., Yoder, O. C., Turgeon, B. G. and Aist, J. R. (1998). A cytoplasmic dynein required for mitotic aster formation in vivo. J. Cell Sci. 111, 2607 2614.

Janmey, P. A. (1991). Mechanical properties of cytoskeletal polymers. Curr. Opin. Cell Biol. 3, 4-11.

Janmey, P. A., Euteneuer, U., Traub, P. and Schliwa, M. (1991). Viscoelastic properties of vimentin compared with other filamentous biopolymer networks. J. Cell Biol. 113, 155-160.

Kake, T., Kimura, S., Takahashi, K. and Maruyama, K. (1995). Calponin induces actin polymerization at low ionic strength and inhibits depolymerization of actin filaments. Biochem. J. 312, 587-592.

Kolakowski, J., Makuch, R., Stepkowski, D. and Dabrowska, R. (1995). Interaction of calponin with actin and its functional implications. Biochem. J. 306, 199-204.

Koonce, M. P., Kohler, J., Neujahr, R., Schwartz, J. M., Tikhonenko, I. and Gerisch, G. (1999). Dynein motor regulation stabilizes interphase microtubule arrays and determines centrosome position. EMBO J. 18, 67866792.

Letourneau, P. C., Shattuck, T. A. and Ressler, A. H. (1987). 'Pull' and 'push' in neurite elongation: observations on the effects of different concentrations of cytochalasin B and taxol. Cell Motil. Cytoskeleton 8, 193209

Ma, S., Trivinos-Lagos, L., Graf, R. and Chisholm, R. L. (1999). Dynein intermediate chain mediated dynein-dynactin interaction is required for interphase microtubule organization and centrosome replication and separation in Dictyostelium. J. Cell Biol. 147, 1261-1274.

Marsh, L. and Letourneau, P. C. (1984). Growth of neurites without filopodial or lamellipodial activity in the presence of cytochalasin B. J. Cell Biol. 99, 2041-2047.

Meberg, P. J. and Bamburg, J. R. (2000). Increase in neurite outgrowth mediated by overexpression of actin depolymerizing factor. J. Neurosci. 20, 2459-2469.

Plantier, M., Fattoum, A., Menn, B., Ben-Ari, Y., Der Terrossian, E. and Represa, A. (1999). Acidic calponin immunoreactivity in postnatal rat brain and cultures: subcellular localization in growth cones, under the plasma membrane and along actin and glial filaments. Eur. J. Neurosci. 11, 28012812.

Winder, S. J., Allen, B. G., Clement-Chomienne, O. and Walsh M. P. (1998). Regulation of smooth muscle actin-myosin interaction and force by calponin. Acta Physiol. Scand. 164, 415-426.

Winder, S. J. and Walsh, M. P. (1996). Calponin. Curr. Top. Cell Regul. 34, 33-61.

Wittmann, T. and Hyman, T. (1999). Recombinant p50/dynamitin as a tool to examine the role of dynactin in intracellular processes. Methods Cell Biol. 61, 37-43

Wu, X., Jung, G. and Hammer, J. A. (2000). Functions of unconventional myosins. Curr. Opin. Cell Biol. 12, 42-51.

Zheng, J., Lamoureux, P., Santiago, V., Dennerll, T., Buxbaum, R. E. and Heidemann, S. R. (1991). Tensile regulation of axonal elongation and initiation. J. Neurosci. 11, 1117-1125. 